\title{
Thermal Emission from a Foam-covered Sea Surface Modelled as a Stratified Layer at Microwave Frequencies
}

\author{
Ayibapreye K. Benjamin*, Aguiyi Nduka Watson **, Godday Biowei*** \\ *Department of Electrical/Electronic Engineering, Niger Delta University \\ ** Department of Electrical/Electronic Engineering, Federal University Otueke \\ **** Department of Electrical/Electronic Engineering Niger Delta University
}

DOI: 10.29322/IJSRP.11.11.2021.p11927

http://dx.doi.org/10.29322/IJSRP.11.11.2021.p11927

\begin{abstract}
In this paper, a physical based model of foam-covered sea surface was modelled as layers of randomly distributed air-bubbles coated with thin layer of sea water which evaluates thermal emission was investigated. Here, the split-step Fourier technique as a range marching procedure is explored by converting the three-dimension (3D) foam structure into two-dimension (2D) layers and determine the vertical field profiles of the propagating E-field at successive range steps until the desired range is reached. The scatterer (foam layer) comprises of randomly distributed packed bubbles with estimated complex dielectric constant of sea foam which is a mixture of air coated with thin layer of sea water. It has internal and external radii $r_{i n}$ and $r_{o}$. The bubble size distribution $N(r)$ follows a lognormal distribution pattern. We consider an arrangement of $N$ bubbles randomly packed closely with no overlap between any two adjacent bubbles. The bubbles are considered to be spherical in shape and are packed in a cubic domain. The inequality $0 \leq z \leq-4 d$ describes the sea foam model in three regions. Results obtained using this method was compared with other experimental results which appear to show a good agreement.
\end{abstract}

Index Terms- Thermal emission, Sea foam, Sea surface, Layered media, Microwave frequencies.

\section{INTRODUCTION}

$\mathrm{C}$ limatologies of the distribution of bubbles in open ocean are based on acoustical and open measurements of bubbles. Global bubble distribution reflects on the dominance of wave breaking to wind speed. Bubbles are an important component of geochemical cycling through their transport of material in the open and surface micro-layer, and especially their role in the air-sea exchange of heat and particles. Hence, there is need to develop a physical based model of foam-covered sea surface that is most suitable for evaluation of millimetre wave scattering and emissivity of sea foam. Wilheit, Pandey and Kakar proposed several empirical models for evaluation of sea foam emissivity and brightness temperature, which was based on experimental data of sea surface by foam, as function of angle of incidence and frequency [1-4]. William asserted that foam emissivity is strongly dependent on foam thickness at X-band in a waveguide [5]. It was demonstrated that foam microstructures and air volume fraction (AVF) are significant in evaluating sea foam emissivity [6], [7]. These factors were further validated by controlled experiments to measure seawater coating thickness, bubble size distribution, and foam layer thickness [8], [9]. The aforementioned empirical models could not explain theoretically, how a foam layer influence microwave radiation. This all-important puzzle necessitates the development of theoretical models which unveil the effects of foam layer to sea surface emission. Theoretical models such as the vector radiative transfer (VTR) equation [10], [11] the effective medium approximation (EMA) theory, and the dense media transfer (DMRT) method were used to investigate emissivity and scattering by sea foam covered sea surface $[12,13,14,15,16,17,18]$. Reasonable results were obtained using these theoretical models but the interactions of air-bubbles coated with thin layer of seawater are not considered precisely in the sea foam complex dielectric constant within millimetre wave by a suitable theory [24]. The effective dielectric constant of a dense media remains a significant parameter in estimating microwave radiation by sea foam. Hence, there is need to develop a more accurate model for estimating the effective dielectric constant of sea foam layer.

Most recently, a systematic insight into the application of various mixing rules (effective medium theories) for evaluating the dielectric constant (permittivity) of sea foam (whitecaps) at microwave frequencies between 1.4 to $37 \mathrm{GHz}$ was reported by [19]. It is significant to note that foam scattering is weak at these frequencies which explains the interest of [19] in evaluating the dielectric constant of whitecaps using inexplicable scattering computations such as Maxwell Garnett, Bruggeman, Coherent potential, Looyenga, and Refractive models. This approach relies on previous findings on various heterogeneous dielectric mixtures and reported characterization 
of sea foam in order to evaluate the availability of various permittivity models for obtaining acceptable predictions of sea foam dielectric constant [26]. Numerous experimental and field observations were presented which characterized deep bubble plumes well but measurements characterizing the surface foam layers are few and usually simulated artificial sea foam [6, 8, 9, 20, 21].

Due to limited knowledge of the microscopic characteristics of sea foam previously published work and experiments, recent models evaluating the foam emissivity and effective permittivity uses exclusively macroscopic foam characteristics, such as void fraction (white- cap coverage) and foam thickness thereby ignoring scattering losses in sea foam. This could be attributed to the fact that previous foam emissivity models that computed the attenuation and permittivity in foam with several scattering theories using as input the microscopic characteristics of foam such as bubble diameters, bubble wall thicknesses, bubble size distribution, filling factor, and stickiness parameter [10], [13] introduced uncertainties which affected the accuracy of the foam emissivity models.

To restrict these uncertainties and enhance use in retrieval algorithms, Bordonskiy et.al suggested the use of exclusively macroscopic quantities instead, namely; void fraction profile and foam layer thickness [26]. Avoiding the use of microscopic quantities implies foregoing modelling of the scattering losses in sea foam. Based on the aforementioned consideration the foam emissivity was obtained by one of the classical permittivity models (mixing rules) which involve only the sea water permittivity $\varepsilon$ [26], air permittivity $\varepsilon_{0}=1-j 0$ and void fraction $f_{a}$. In [22] the foam layer was assumed to be much thicker than the penetration depth of the radiation, thus ignoring the emissivity of the water below the foam layer. Evaluations of extinction, scattering and absorption coefficients were carried out at $19.35 \mathrm{GHz}$ for foam consisting of large bubbles (inner radius of $4.3 \mathrm{~mm}$ ) with relatively thick walls (0.13 mm). Zhang et.al obtained results which represents a numerical analogue of Williams experimental observations for foam spread over an aluminium plate. The model in [22] eliminated a major contributor to absorption (the water below the foam) and considered large thick- walled bubbles at relatively high frequency. This unsurprisingly, predicts low absorption (about 28\% of the total extinction) and significant scattering, which appear consistent with the other analytical and experimental findings [26].

\section{SCATTERING AND EMISSION BY LAYERED MEDIA}

Sea foam layer was modelled as a layered medium with reflective boundaries. Here we investigate thermal emission from these layers in the form of sea surface emissivity and brightness temperature using coherent method. The coherent method is derived from Maxwell's equation and the fluctuation dissipation theorem [23].

\section{Emissivity}

Emissivity is expressed as the ratio of the emissive power of the particle to the emissive power of a blackbody of the same size and shape at the same constant temperature. Expressly, the emissivity describes the emissive power of an arbitrary opaque body of fixed shape and size at a definite temperature.

\section{Brightness Temperature}

Observed responses (thermal radiation emitted or scattered) due to inter- action of incident EM waves with unique polarizations and physical objects on the earth surface or atmosphere from a vintage point to the observer are measured by satellite radiometer. Brightness temperature of a surface is measured by satellite radiometers by thermal radiation emitted from the surface due to interaction between incident EM waves and particles or objects in the earth surface or atmosphere.

Radiance temperature or brightness temperature measures microwave radiation travelling upward from the top of the atmosphere to the satellite, given in units of an equivalent blackbody temperature. Passive microwave radiometers measure the brightness temperature as a fundamental parameter used by remote sensing systems (RSS) to derive wind, vapour, rain, cloud and sea surface temperature (SST) products. In spite of variation in sensor frequencies, resolutions in channels, instrument operation and other radiometer characteristics, RSS produces high quality, carefully inter-calibrated data, using uniform processing techniques, using uniform processing techniques, with a brightness temperature data record spanning multiple instruments over several decades. Brightness temperature characterizes radiation and differs considerably from physical temperature of a radiating body due to the mechanism of radiation. However, it is feasible to construct a device which will heat up a source of radiation with some $\mathrm{T}_{\mathrm{B}}$ equal to the actual temperature of the body. $\mathrm{T}_{\mathrm{B}}$ as ordinarily understood is not a temperature but typically obtained by an optical measurement using a pyrometer with the purpose of evaluating the real temperature of a surface. As shown below that the real temperature of a surface can be obtained by the ratio of the brightness temperature and emissivity of the surface.

$$
T_{B}=T_{s} e_{p}^{\text {foam }}
$$


Emissivity lies between 0 and 1 and real temperature of a body will be greater or equal to the brightness temperature. For higher frequencies (short wavelength) and low temperatures, conversion of these parameters must obey Planck's law.

\section{COHERENT METHOD}

The coherent or wave method was used to solve for the reflective and transmitted wave amplitudes by the sea foam layer. The incident polarization is expressed as a linear combination of the horizontal (TE) and vertical (TM) polarizations. Amplitudes of propagating waves in each layer can be obtained by propagating matrices, and the reflection coefficient is expressed in a recurrence relation. The foam layer is a stratified medium with boundaries The foam layer is a stratified medium with boundaries $z=0 \ldots$.., $-4 d$. (Figure.1). The $(n+1)$ th region is semi-infinite and labelled $t, t=n+1$. In any region $l$, the medium is characterized by its complex permittivity $\varepsilon_{l}$ with permeability $\mu_{l}=1$. An incident plane wave impinges on the stratified medium; the transverse electric (TE) and transverse magnetic (TM) polarizations are treated independently. The z-axis and incident vector $\bar{k}$ determines the plane of incidence [23].

Maxwell's equations were used to express the electromagnetic (EM) field components for a horizontally polarized incident wave in terms of a single component $H_{l}$ in any region $l$.

$$
\begin{aligned}
& \left(\nabla_{\perp}^{2}+k_{l}^{2}-k_{l z}^{2}\right)=0 \\
& \overline{H_{l \perp}}=\frac{1}{k_{l}^{2}-k_{l z}^{2}} \nabla_{\perp}\left[\frac{\partial}{\partial z} H_{l z}\right] \\
& \overline{E_{l \perp}}=\frac{i \omega \mu_{l}}{k_{l}^{2}-k_{l z}^{2}} \times \nabla_{\perp}\left[\bar{z} H_{l z}\right]
\end{aligned}
$$

where $k_{l}=\omega \sqrt{\mu_{l} \varepsilon_{l}}, k_{l z}=\sqrt{k_{l}^{2}-\left|\overline{k_{\perp}}\right|^{2}}, \overline{k_{\perp}}$ is the incident wave vector $\bar{k}$ component in the $x-y$ plane. $\nabla_{\perp}$ is the two dimensional del operator and $\nabla_{\perp}^{2}$ is two-dimensional Laplacian operator in the $x-y$ plane. The incident TE wave on the sea foam layer is given as $H_{z}=H_{o} e^{i k_{z} z} e^{i \overline{k_{\perp}} \cdot \overline{r_{\perp}}}$ and the total field in the region $l$ can be expressed as

$$
H_{l z}=\left(A_{l} e^{i k_{l z} z}+B_{l} e^{i k_{l z} z}\right) e^{i \overline{k_{\perp}} \cdot \overline{r_{\perp}}}
$$

$\overline{k_{\perp}}$ doesn't have the subscript $l$ because $\overline{k_{\perp}}$ values are equal in all regions due to phase matching. Taking region 0 where $l=0$, the field amplitude for the backward and forward travelling waves are expressed as

$$
\begin{gathered}
A_{0}=R H_{0} \\
B_{0}=H_{0}
\end{gathered}
$$

$\mathrm{R}$ and $T$ are reflection and transmission coefficients respectively. In the region $t$, where $l=n+1$, we have

$$
\begin{array}{r}
A_{0}=0 \\
B_{t}=T H_{0}
\end{array}
$$

$\mathrm{R}$ is derived from the boundary conditions. When the interface $z=d_{l}$ which is the boundary between $l$ and $l+1$ regions, the tangential $\overline{E_{l \perp}}$ and $\overline{H_{l \perp}}$ fields are continuous and expressed below. 


$$
\begin{array}{r}
k_{l z}\left(A_{l} e^{-i k_{l z} d_{l}}-B_{l} e^{i k_{l z} d_{l}}\right)=k_{(l+1) z}\left(A_{l+1} e^{-i k_{(l+1) z} d_{l}}-B_{l+1} e^{i k_{(l+1) z} d_{l}}\right) \\
\mu_{l}\left(A_{l} e^{-i k_{l z} d_{l}}-B_{l} e^{i k_{l z} d_{l}}\right)=\mu_{(l+1) z}\left(A_{l+1} e^{-i k_{(l+1) z} d_{l}}-B_{l+1} e^{i k_{(l+1) z} d_{l}}\right)
\end{array}
$$

For $n+1$ boundaries, there are two equations at each boundary as shown in 10 and 11 . Hence, there are $2 n+2$ equations to solve for $2 n+2$ unknowns $A_{l}$ and $B_{l}, l=1,2 \ldots, n$ and $A_{0}$ and $B_{l}$. Expressing $A_{l}$ and $B_{l}$ in terms of $A_{l+1}$ and $B_{l+1}$, we obtain

$$
\begin{gathered}
A_{l} e^{-i k_{l z} d_{l}}=\frac{1}{2}\left\{\frac{\mu_{(l+1)}}{\mu_{l}}+\frac{k_{(l+1) z}}{k_{l z}}\right\}\left(A_{l+1} e^{-i k_{(l+1) z} d_{l}}-R_{l(l+1)} B_{l+1} e^{i k_{(l+1) z} d_{l}}\right) \\
B_{l} e^{-i k_{l z} d_{l}}=\frac{1}{2}\left\{\frac{\mu_{(l+1)}}{\mu_{l}}+\frac{k_{(l+1) z}}{k_{l z}}\right\} R_{l(l+1)}\left(A_{l+1} e^{-i k_{(l+1) z} d_{l}}-B_{l+1} e^{\left.i k_{(l+1) z} d_{l}\right)}\right. \\
R_{l(l+1)}=\frac{\mu_{(l+1)} k_{l z}-\mu_{l} k_{(l+1) z}}{\mu_{l+1} k_{l z}+\mu_{l} k_{(l+1) z}} \\
\frac{A_{l}}{B_{l}} e^{-i 2 k_{l z} d_{l}}=\frac{\frac{A_{l+1}}{B_{l+1}} e^{-i 2 k_{(l+1) z} d_{l+1}} e^{i 2 k_{(l+1) z}\left(d_{l+1}-d_{l}\right)}+R_{l(l+1)}}{\frac{A_{l+1}}{B_{l+1}} e^{-i 2 k_{(l+1) z} d_{l+1} R_{l(l+1)} e^{i 2 k_{(l+1) z}\left(d_{l+1}-d_{l}\right)}+1}}
\end{gathered}
$$

For the last layer $\frac{A_{t}}{B_{t}}=0$, equation (15) is a recurrence relation that describes $\frac{A_{l}}{B_{l}} e^{-i 2 k_{l z} d_{l}}$ in terms of $\frac{A_{l+1}}{B_{l+1}}$. We can express $e^{i 2 k_{(l+1) z} d_{l+1}}$ in terms of $\frac{A_{l+2}}{B_{l+2}}, e^{i 2 k_{(l+1) z} d_{l+1}}$ and so on until the transmission region $t$ is reached where $\frac{A_{t}}{B_{t}}=0$. The reflection coefficient for the stratified foam layer $R_{h}=\frac{A_{0}}{B_{0}}$ can be evaluated using the approach below. Consider $\frac{A_{0}}{B_{0}}=0$ and $t=n+1$, by adapting equation (15), we compute $\frac{A_{n-1}}{B_{n-1}} e^{i 2 k_{(n-1) z} d_{n-1}}$ and so on, until the zeroth region is reached and $R_{h}=\frac{A_{0}}{B_{0}}$ is computed. The reflection coefficient for TM wave is obtained by duality. It was achieved by replacing $\mu_{l} \rightarrow \varepsilon_{l}, \varepsilon_{l} \rightarrow \mu_{l}, \bar{H}_{l} \rightarrow \bar{E}_{l}$, and $\bar{E}_{l} \rightarrow$ $\bar{H}_{l}[23]$.

\section{COMPLEX EFFECTIVE DIELECTRIC CONSTANTS OF STRATIFIED SEA FOAM LAYERS}

The adopted approach models sea foam to account for the optical and geometrical properties such as foam layer thickness, foam void fraction, bubble radii, bubble size distribution (BSD), and bubble shape. BSD and bubble radii are computed by inverse cumulative error function using Newton Raphson method. Random sphere close packing is applied for filling the bubbles in three-dimensional (3D) domain [24]. The domain could be cube, cuboid, prism, or cylinder but a cubic domain was assumed for simplicity as we in- tend to divide the (3D) domain into slices of 2D domain [24]. The packing of air-bubbles or spheres is done such that no two adjacent bubbles overlap. For irregular distribution of air-bubbles (foams), the random close packing is used to achieve a densely packing with bubble volume fraction of $64 \%$, with the within the host domain having a geometry of $36 \%$ for maximum packing density [24].

In this approach the air-bubbles locations or positions were generated using uniformly distributed random numbers $n=100,000$ in $X$, $\mathrm{Y}$ and $\mathrm{Z}$ coordinates. Computation of bubble radii for n-randomly distributed bubbles were done in FORTRAN 90 complier using inverse method. The inverse method transforms uniform variate $U(0,1)$ to normal variate $N(0,1)$. BSD $N(r)$ and bubble radii $r_{i}$ were computed with mean $\mu=2.0$ and standard deviation $\sigma=0.5$, geometric mean $\mu_{g}=500$ micron and geometric standard deviation $\sigma_{g}=2.0$. This was implemented using an empirical expression for the scaled normal variate and log-normally distributed BSD [24].

The Split-step Pad'e approximation with finite differencing was adopted for evaluation of thermal emission in the foam layered sea surface. The Split- step Fourier method is a range marching technique efficient for prediction of field perturbations at each slice of foam layer. Hence, the need to translate 3-D to 2-D slices [24]. 


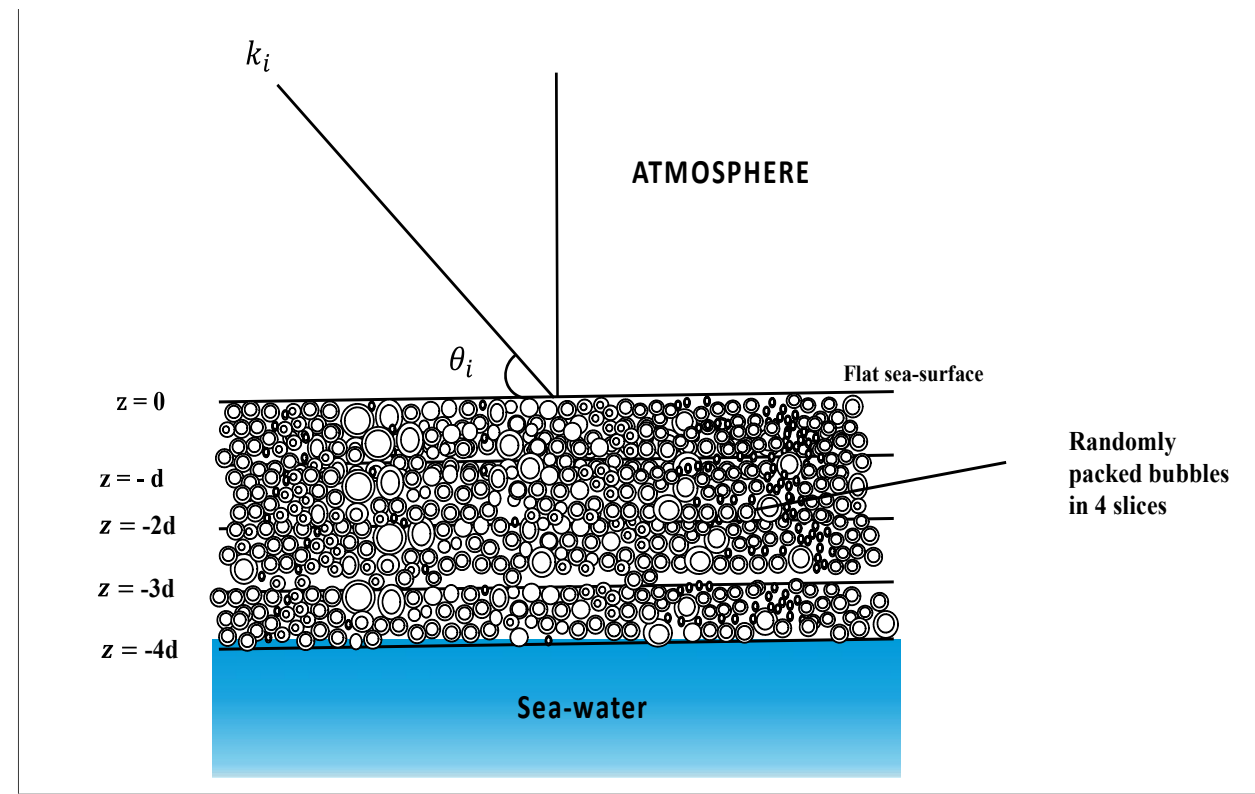

Figure 1. Geometrical configuration of foam-covered sea surface shows thermal emission from the flat-sea surface. The foam layer region is divided into 5 slices or layers which are equidistant and accounts for scattering and absorption of millimetre wave at WindSat frequencies [24].

For fixed salinity $(34 \mathrm{psu})$, sea surface temperature $\left(20^{\circ} \mathrm{C}\right)$ and frequency range between $1.4 \mathrm{GHz}$ to $37.0 \mathrm{GHz}$, the dielectric constant of sea water was calculated by adopting existing methods by Stogryn, Guillou, Wentz, English, Klein and Swift [24]. These calculated dielectric constants were used to estimate the effective permittivity of sea foam. The area of the circles in each slice was calculated using the total number of grid points [26].

The effective dielectric constants of sea foams at frequencies $10.7 \mathrm{GHz}$ and $37.0 \mathrm{GHz}$ were calculated for 5 slices of randomly packed air-bubbles coated with thin layer of seawater [26].

\begin{tabular}{|c|c|c|}
\hline FREQUENCY & $10.7 \mathrm{GHZ}$ & $37 \mathrm{GHz}$ \\
\hline Slice 1 & $1.0948-0.1251 \mathrm{i}$ & $1.0006-0.0332 \mathrm{i}$ \\
\hline Slice 2 & $1.1248-0.1507 \mathrm{i}$ & $1.0108-0.0239 \mathrm{i}$ \\
\hline Slice 3 & $1.1622-0.1810 \mathrm{i}$ & $1.0225-0.0344 \mathrm{i}$ \\
\hline Slice 4 & $1.1983-0.2072 \mathrm{i}$ & $1.0315-0.0569 \mathrm{i}$ \\
\hline Slice 5 & $1.2271-0.2277 \mathrm{i}$ & $1.0465-0.0637 \mathrm{i}$ \\
\hline
\end{tabular}

Table 1. Results for Dielectric constant of sea foam at frequencies of $10.7 \mathrm{GHz}$ and $37 \mathrm{GHz}$ for 5 2-D Slices of randomly packed airbubbles covered with thin-layer of seawater [24],[26].

The effective dielectric constant of sea foam increases with increase in thickness of foam layer and decreases with increase in frequency as illustrated in Table 1. The effective dielectric constant of sea foam is used in the computation of Fresnel's reflection coefficients for both horizontal $R_{p}^{\text {foam }}$ and vertical $R_{v}^{\text {foam }}$ polarized fields, at the air-foam interface and the foam-ocean interface [23]. The Fresnel's reflection coefficients are used for the computation of sea surface emissivity $e^{\text {foam }}$ and brightness temperature $T_{B}$ in the radiative transfer equations given below.

$$
\begin{aligned}
& e_{p}^{\text {foam }}=1-\left|R_{p}^{\text {foam }}\right|^{2} \\
& e_{v}{ }^{\text {foam }}=1-\left|R_{v}^{\text {foam }}\right|^{2}
\end{aligned}
$$


where $e_{p}^{\text {foam }}$ is the sea surface emissivity, $p=h$ (horizontal polarization) and $v=s$ for vertical polarization.

$$
\begin{gathered}
T_{B}=e_{p}{ }^{\text {foam }} T_{s} \\
T_{B}=e_{v}{ }^{\text {foam }} T_{S}
\end{gathered}
$$

where $T_{S}$ denote sea surface temperature. The expressions of reflection coefficients for multi-layered foam structure for both horizontal $R_{p}^{\text {foam }}$ and vertical $R_{v}^{\text {foam }}$ polarized fields are given in [23].

$R_{p}$ is the reflection coefficient of the foam layer which describes the amplitudes of a reflected wave relative to an incident wave and is closely related to the transmission coefficient, For the slices of sea foam layer which exemplifies multi-layered composites each layer having different dielectric constants, there are multiple reflections and transmission in each layer caused by the boundary separating of different regions [23].

The reflection coefficient $R_{p}$ reflects the effect of all transmission and reflection of an incident wave in the stratified medium and its expression for multi-layers are shown in [23].

\section{RESULTS AND FINDINGS}




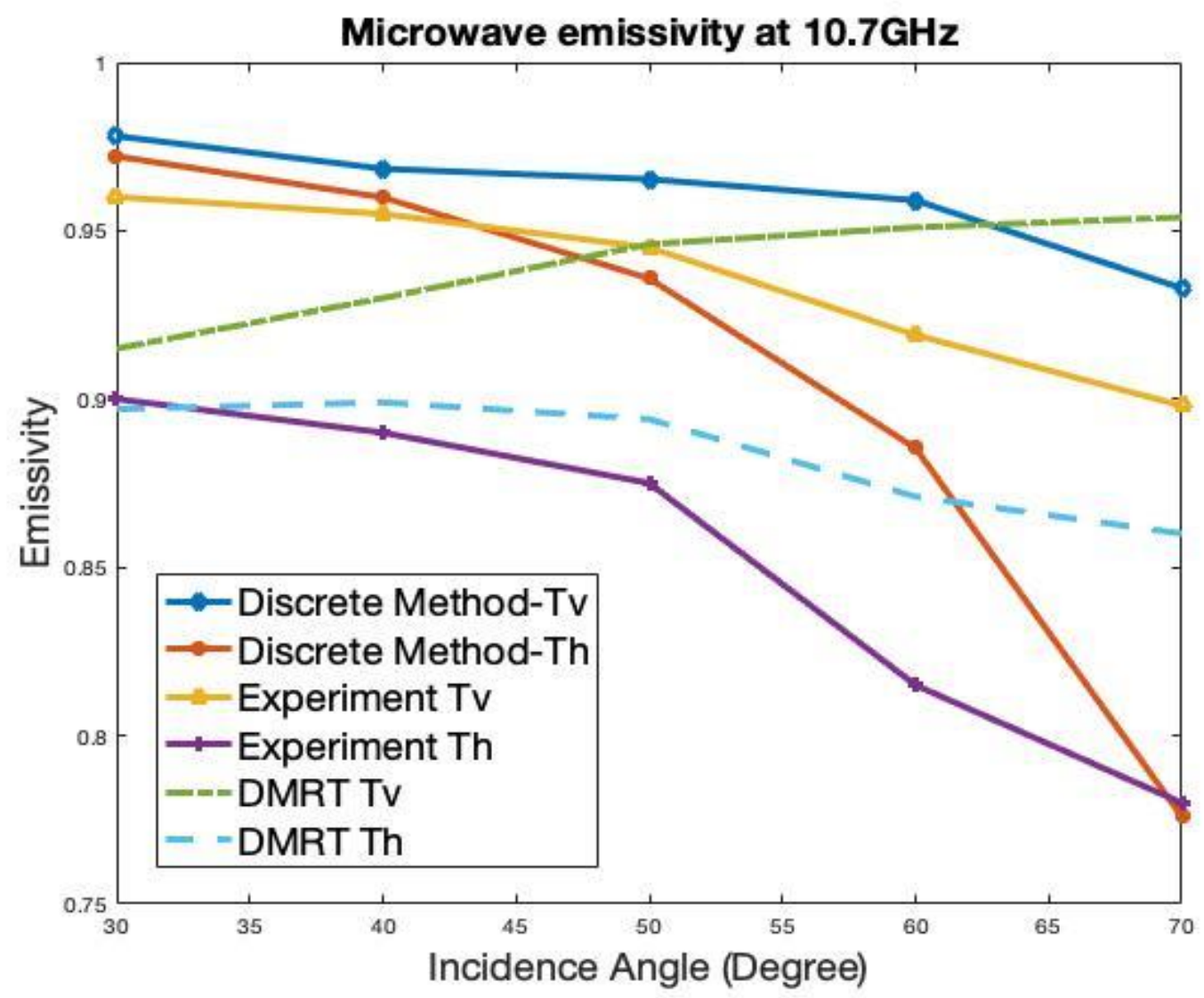

Figure 2. Emissivity of Sea surface by Foam as a Function of Incident angle at $10 \mathrm{GHz}$ compared with Dense Media Radiative Transfer (DMRT) and Experimental method.

For incident angles $\theta_{i}=30^{\circ}, 35^{\circ}$ and $40^{\circ}$, Figures 2 and 3 show the emissivity variations at $10.7 \mathrm{GHz}$ and $37 \mathrm{GHz}$ for TE and TM polarizations. These were compared to Experimental method and DMRT. The emissivity as the ratio of the emissive power of the particle to the emissive power of a blackbody of the same size and shape at the same constant temperature from the foam-covered sea surface decreases with increase in incident angle. $T_{B}$ for Experimental methods falls in similar manner as that of the discrete method for vertical polarization. $T_{B}$ increases rapidly for incident angles between $30^{\circ}$ and $50^{\circ}$ and steadily for angles between $50^{\circ}$ and $70^{\circ}$ for DMRT Method when vertically polarized. $T_{B}$ for horizontally polarized DMRT method shows it increases slowly between $30^{\circ}$ and $50^{\circ}$ and decreases gradually between $50^{\circ}$ and $70^{\circ}$. Discrete method and DMRT follows similar pattern as they decrease from $30^{\circ}$ and $70^{\circ}$ for TE mode. 


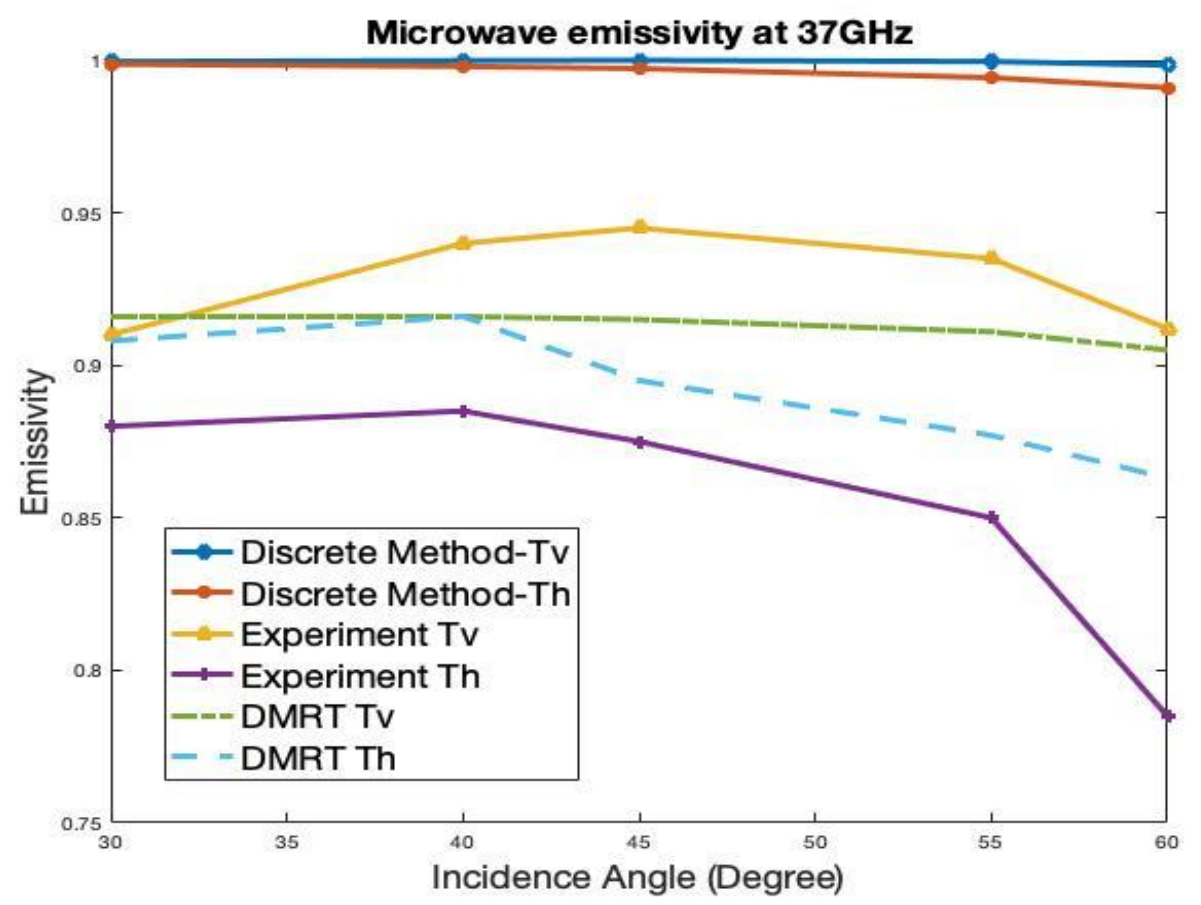

Figure 3. Emissivity of Sea Surface Covered by Foam as a Function of Incident Angle at $37 \mathrm{GHz}$ Compared with DMRT and Experimental Method.

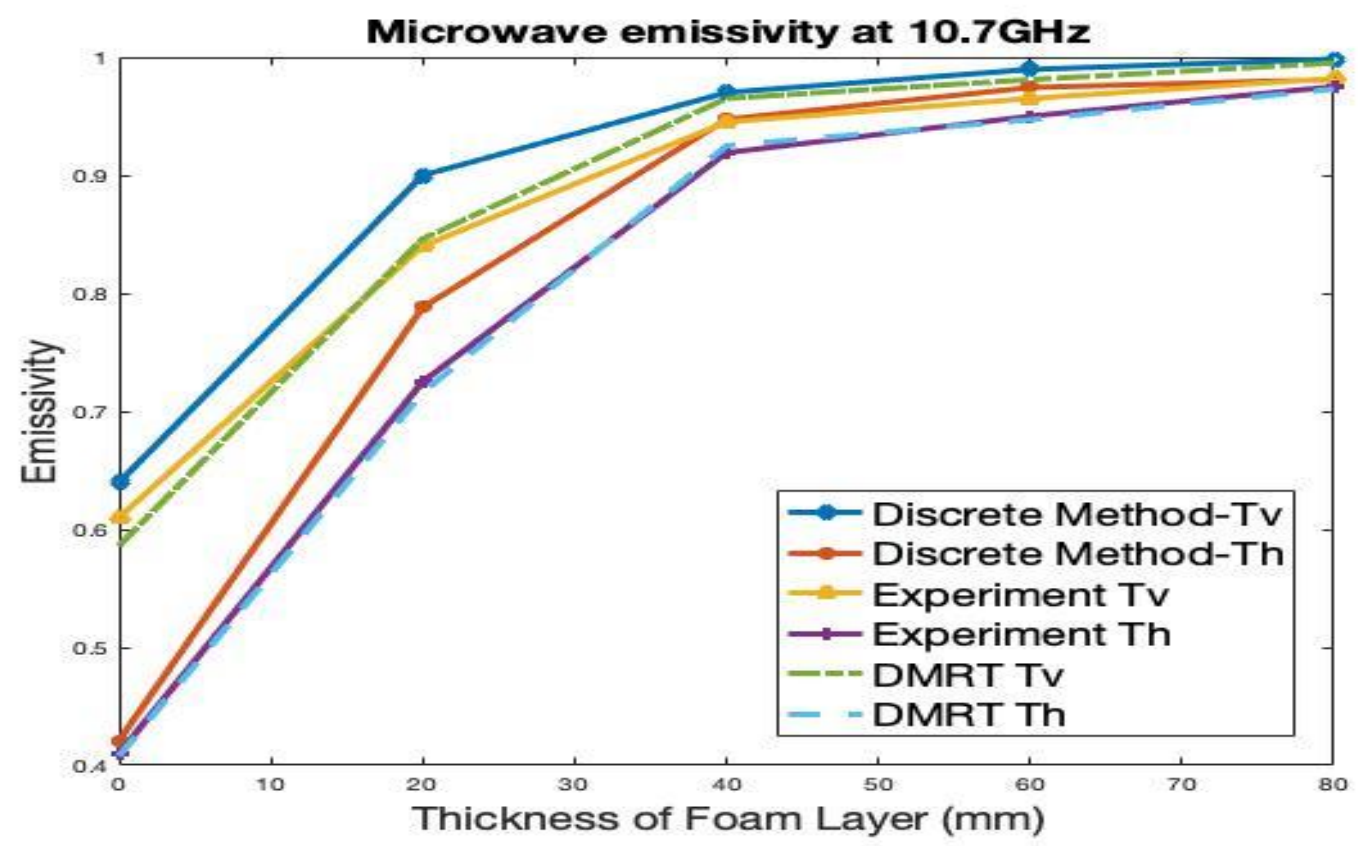

Figure 4. Emissivity of Sea Surface Covered by Foam as a Function of Foam Layer Thickness at $10.7 \mathrm{GHz}$ Compared with DMRT and Experimental Method.

Figures 4 and 5 show plots of microwave emissivity dependence on foam layer thickness at $10.7 \mathrm{GHz}$ and $37 \mathrm{GHz}$ respectively, for both vertical and horizontal polarizations. These show that foam emissivity using experimental data, increases at angles with increase in foam layer thickness for both horizontal and vertical polarizations. The discrete method correspondingly, increases with increase in angle of incidence for both horizontal and vertical polarizations at $10.7 \mathrm{GHz}$ and $37 \mathrm{GHz}$. The (DMRT) dense media radiative transfer yield results in reasonably good agreement with experimental measurements for both horizontal and vertical polarizations. The results show 
comparable emissivities at $10.7 \mathrm{GHz}$ and $37 \mathrm{GHz}$. Absorption effect at $37 \mathrm{GHz}$ is larger than $10.7 \mathrm{GHz}$ while scattering effect is more significant at $37 \mathrm{GHz}$. The results are in good agreement at all incidence angles

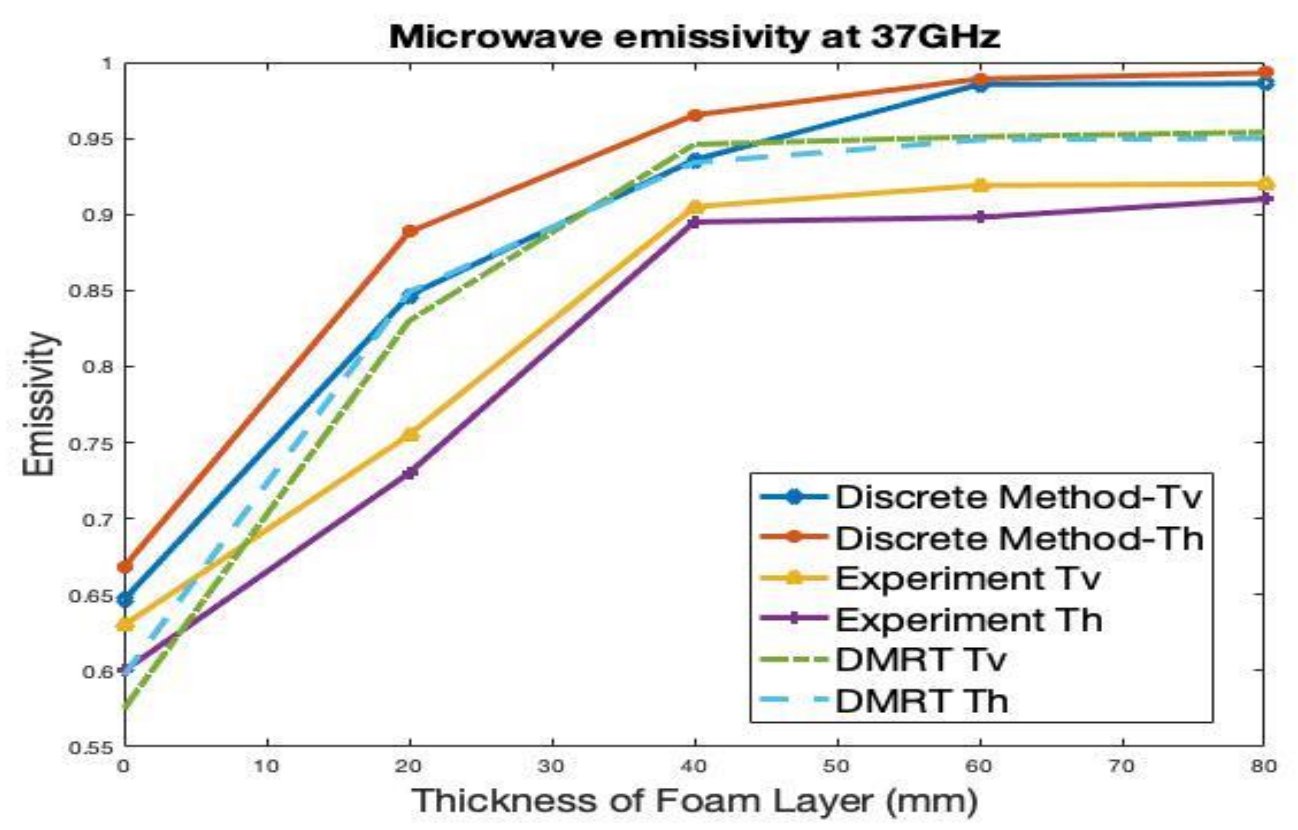

Figure 4. Emissivity of Sea Surface Covered by Foam as a Function of Foam Layer Thickness at $37 \mathrm{GHz}$ Compared with DMRT and Experimental Method.

\section{CONCLUSION}

For fixed salinity, sea surface temperature and frequencies $10.7 \mathrm{GHz}$ and $37 \mathrm{GHz}$, the dielectric constant of sea water was calculated by adopting existing methods by Stogryn, Guillou, Wentz, English, Klein and Swift. These calculated dielectric constants were used to estimate the effective permittivity of sea foam. The effective dielectric constants of sea foams at frequencies $10.7 \mathrm{GHz}$ and $37.0 \mathrm{GHz}$ were calculated for 5 slices of randomly packed air-bubbles coated with thin layer of seawater. Table 1 shows variation of effective dielectric constant at $10.7 \mathrm{GHz}$ and $37 \mathrm{GHz}$ for 5 2-D Slices of randomly packed air-bubbles covered with thin-layer of seawater. The effective dielectric constant of sea foam increases with increase in thickness of foam layer and decreases with increase in frequency as illustrated in Table 1. The effective dielectric constant of sea foam is used in the computation of Fresnel's reflection coefficients for both horizontal and vertical polarized fields, at the air-foam interface and the foam-ocean interface. The Fresnel's reflection coefficients are used for the computation of sea surface emissivity and brightness temperature in the radiative transfer equations given in equation $16-19$.

\section{Acknowledgment}

This research work was supported financially Dr. Nimibofa Ayawei. 


\section{References}

[1] T.T. Wilheit Jr, "A model for the microwave emissivity of the ocean's surface as a function of wind speed," IEEE Trans. Geosci. Electron., vol. GE-17, pp. 244249, April 1979.

[2] P.C. Pandey and R. K. Kakar, “An empirical microwave emissivity model for a foam-covered sea,” IEEE J. Oceanic Eng., vol. OE-7, no.3, pp. 135-140, 1982

[3] P. M. Smith, "The emissivity of sea foam at 19 and 37GHz," IEEE Trans. Geosci. Remote Sensing, vol. 26, pp. 541-547, Sept. 1998.

[4] A. Stogryn, "The emissivity of sea foam at microwave frequencies," Journal of Geophysical Research, vol. 77, no. 9, pp. 1658-1666, 1972.

[5] G. F. Williams, "Microwave emissivity measurements of bubbles and foam,” IEEE Trans. Geosci. Electron., vol. GE-9, pp. $221-244$, July 1971.

[6] Y. A. Militskii et.al. “Thermal radio emission from foam structures,” Sov. Phy. Tech. Phys. Engl. Transl. vol 23, pp. 601-602, 1978.

[7] J. Podzimek, "Size spectra of bubbles in the foam patches and sea salt nuclei over the surf zone," Tellus 36 B(3), pp. 192-202, 1984.

[8] L. A. Rose, W. E. Asher, S. C. Reising, P. W. Gaiser, K. M. St Germain, D. J. Dowgiallo, K. A. Horgan, G. Farquharson, and E. Knapp, "Radiometric measurements of the microwave emissivity of foam," Geoscience and Remote Sensing, IEEE Transactions on, vol. 40, no. 12, pp. 2619-2625, 2002.

[9] A. Camps, M. Vall-Llossera, R. Villarino, N. Reul, B. Chapron, I. Corbella, N. Duffo, F. Torres, J. J. Miranda, R. Sabia, et al., "The emissivity of foam-covered water surface at L-band: Theoretical modelling and experimental results from the frog 2003 field experiment," Geoscience and Remote Sensing, IEEE Transactions on, vol. 43, no. 5, pp. 925-937, 2005.

[10] D. Chen, L. Tsang, L. Zhou, S. C. Reising, W. E. Asher, L. A. Rose, K.-H. Ding, and C.- T. Chen, "Microwave emission and scattering of foam based on MonteCarlo simulations of dense media," Geoscience and Remote Sensing, IEEE Transactions on, vol. 41, no. 4, pp. 782-790, 2003.

[11] X. Z. Huang and Y. Q. Jin, "Scattering and emission from two-scale randomly rough sea surface with foam scatterers," Proc. Inst. Elect. Eng. Microwave Antenna Propagat., vol. 142, pp. 109-114, April 1995.

[12] L. Tsang, C-T. Chen, A. T. C. Chang, J. Guo, and K. H. Ding, "Dense media radiative transfer based on quasi-crystalline approximation with applications to passive microwave remote sensing of snow," Radio Sci., vol. 35, no. 3, pp. 731-749, 2000.

[13] J. Guo, L. Tsang, W. Asher, K.-H. Ding, and C.-T. Chen, "Applications of dense media radiative transfer theory for passive microwave remote sensing of foamcovered ocean," Geoscience and Remote Sensing, IEEE Transactions on, vol. 39, no. 5, pp. 1019-1027, May 2001.

[14] Wei, E. B., and Ge, Y., “A microwave emissivity model of sea surface under wave breaking,” Chinese Physics, vol. 34, no. 6, pp. 1259-1264, 2005.

[15] Lui, S. B., Wei, E. B., Hong, J. L., and Ge, Y., "Microwave backscattering from the sea surface with breaking waves," Chinese Physics, vol. 15, no. 9, pp. 21752179,2006

[16] Wei, E. B., and Liu, Y., “Application of effective medium approximation theory to ocean remote sensing under wave breaking," Science in China Series D: Earth Science, vol. 50, no. 3, pp. 474-480, 2007.

[17] Wei, E. B., "Microwave vector radiative transfer equation of a sea foam layer by the second-order Rayleigh approximation," Radio Science, vol. 46, RS5012, 2011.

[18] Wei, E. B., "Effective medium approximation model of sea foam layer microwave emissivity of a vertical profile," International Journal of Remote Sensing, vol. 34, no. 4, pp. 1180-1193, 2013.

[19] M. D. Anguelova, "Complex dielectric constant of sea foam at microwave frequencies," Journal of Geophysical Research: Oceans (1978-2012), vol. 113, no. C8, 2008.

[20] I. MILITSKII, V. RAIZER, E. Sharkov, and V. Etkin, "Scattering of microwave radiation by foamy structures (honeycomb and emulsion type sea surfs)," Radio Engineering and Electronic Physics, vol. 22, pp. 46-50, 1977.

[21] R. D. Peltzer and O. M. Griffin, "Stability of a three-dimensional foam layer in seawater," Journal of Geophysical Research: Oceans (1978-2012), vol. 93, no. C9, pp. 10804-10812, 1988.

[22] Y. Zhang, Y. Yang, and J. Kong, "A composite model for estimation of polarimetric thermal emission from foam covered wind-driven ocean surface," Prog. Electromagn. Res., vol. 37, pp. 143-190, 2002.

[23] Tsang, Leung, Jin Au Kong, and Kung-Hau Ding. Scattering of electromagnetic waves: theories and applications. Vol. 27. John Wiley \& Sons, 2004.

[24] Benjamin, Ayibapreye K., and Collins E. Ouserigha. "Computation of Brightness Temperature of Sea-foam Modelled as Sequences of Thin Phase Screens using Matlab", European Scientific Journal, vol. 16, no. 3, pp 1857-7431, January 2020.

[25] BENJAMIN, Ayibapreye Kelvin, and Collins E. OUSERIGHA. "Extinction of Millimeter wave on Two Dimensional Slices of Foam-Covered Sea-surface.", International Journal of Scientific Research and Engineering Development, vol. 3, no. 4, July-August 2020.1

[26] Ayibapreye Kelvin Benjamin, "Evaluation of Sea-foam Effective Dielectric Constant at Microwave Frequencies using Maxwell Garnett's and Bruggeman's Methods", International Journal of Scientific Research and Engineering Development, vol. 3, no. 5, September 2020. 


\section{Authors}

First Author - Ayibapreye Kelvin Benjamin, Ph. D in Computing and Electronic Systems University of Essex, United Kingdom, M.Sc. in Electronic Communications and Computer Engineering, The University of Nottingham, United Kingdom, B.Eng. Electrical/Electronic Engineering, Niger Delta University, Nigeria, Niger Delta University, Wilberforce Island Bayelsa State, Nigeria. ayibapreyebenjamin@ndu.edu.ng

Second Author - Aguiyi Nduka Watson, M.Eng. in Electrical Engineering, Rivers State University, Nigeria, B.Eng. Electrical Engineering, Rivers State University, Nigeria, Federal University Otueke, Bayelsa State, Nigeria. aguiyiwatson@ gmail.com

Third Author - Godday Biowei, Ph.D Candidate in the Department of Niger Delta University, Electrical/Electronic Engineering, Wilberforce Island Bayelsa State, Nigeria, M.Sc. in Electronic Communications and Computer Engineering, The University of Nottingham, United Kingdom, B.Eng. Electrical/Electronic Engineering, Niger Delta University, Nigeria

Correspondence Author - Ayibapreye Kelvin Benjamin, Ph. D in Computing and Electronic Systems University of Essex, United Kingdom, M.Sc. in Electronic Communications and Computer Engineering, The University of Nottingham, United Kingdom, B.Eng. Electrical/Electronic Engineering, Niger Delta University, Nigeria, Niger Delta University, Wilberforce Island Bayelsa State, Nigeria. ayibapreyebenjamin@ndu.edu.ng, contact number: +2348166924395. 